\title{
Avaliação do método de espectrometria por cintilação em meio líquido para a medida das atividades alfa e beta total em água: aplicação a águas de abastecimento público no estado de Goiás, Brasil
}

\author{
Evaluation of the liquid scintillation spectrometry method for \\ measurement of the gross alpha and beta activities in water: \\ application to drinking water from Goiás state, Brazil
}

Raquel Maia Mingote', Heliana Ferreira da Costa ${ }^{2}$

\begin{abstract}
RESUMO
Uma revisão da determinação de alfa e beta total em água, usando espectrometria de cintilação em meio líquido, éapresentada. Foram identificados os principais fatores que influenciam a exatidão e a repetibilidade dos resultados analíticos: pré-concentração térmica, tipo de ácido, janela de contagem e padrão de calibração. Um procedimento analítico foi estabelecido e aplicado a amostras de água de abastecimento público do estado de Goiás para avaliação das radioatividades alfa e beta total. O resumo estatístico dos dados foi realizado por meio dos métodos de análise de dados censurados. Os resultados estão consistentes com os valores de referência do Ministério da Saúde.

Palavras-chave: espectrometria de cintilação em meio líquido; alfa total; beta total; água
\end{abstract}

\begin{abstract}
A review to determine the gross alpha and beta in water by using liquid scintillation spectrometry is presented. The main factors that influence the accuracy and repeatability of the analytical results are thermal pre-concentration, acid type, counting window, and calibration standard. Accordingly, an analytical procedure was established and carried out to samples of the public water supplies in the state of Goiás, Brazil, to evaluate the gross alpha and beta radioactivities. The statistical summary of data was conducted by using analysis methods of censored data. The results comply with the guideline values from the Brazilian Department of Health.
\end{abstract}

Keywords: liquid scintillation counting; gross alpha; gross beta; water

\section{INTRODUÇÃO}

A Portaria 2.914/2011 do Ministério da Saúde (BRASIL, 2011) dispõe sobre a qualidade da água para consumo humano e estabelece como níveis de triagem, que conferem a potabilidade da água do ponto de vista radiológico, valores de concentração que não excedam $0,5 \mathrm{~Bq} \cdot \mathrm{L}^{-1}$ para alfa total e 1 Bq. $\mathrm{L}^{-1}$ para beta total. Caso eles sejam superados, deve ser feita a análise dos radionuclídeos presentes. Do ponto de vista radiológico, são considerados ${ }^{226} \mathrm{Ra} \mathrm{e}^{228} \mathrm{Ra}$ produtos das séries de decaimento natural do ${ }^{238} \mathrm{U}$ e ${ }^{232} \mathrm{Th}$, respectivamente. O resultado deve ser comparado com os níveis de referência de $1 \mathrm{~Bq} \cdot \mathrm{L}^{-1}$ para ${ }^{226} \mathrm{Ra}$ e 0,1 Bq. $L^{-1}$ para ${ }^{228} \mathrm{Ra}$.

Rádio, urânio $\mathrm{e}^{40} \mathrm{~K}$, este último um radioisótopo do potássio de ocorrência natural, são os radionuclídeos com mais possibilidades de serem encontrados em águas para consumo humano. O potássio está amplamente distribuído no ambiente, incluindo todas as fontes naturais de água, e pode ocorrer na água tratada em função do uso de permanganato de potássio como oxidante no tratamento da água. As concentrações de potássio normalmente encontradas na água para consumo humano são baixas e não representam risco à saúde (WHO, 2011). Considerando

'Mestre em Ciências e Técnicas Nucleares, Centro de Desenvolvimento da Tecnologia Nuclear (CDTN) - Belo Horizonte (MG), Brasil.

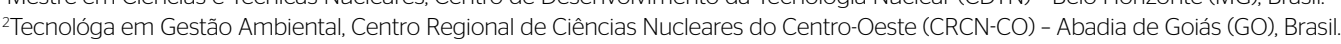

Endereço para correspondência: Raquel Maia Mingote - Centro de Desenvolvimento da Tecnologia Nuclear (CDTN), Comissão Nacional de Energia Nuclear (CNEN) - Caixa Postal 941- 30161-970 - Belo Horizonte (MG), Brasil - E-mail: raquel.mingote@cdtn.br

Recebido: 22/10/14 - Aceito: 27/01/16 - Reg. ABES: 141973 
que, do ponto de vista de risco à saúde, são mais significantes os aspectos de toxicidade química do urânio para os rins, o urânio é classificado como um contaminante químico, e não radiológico. É estabelecida em 0,03 mg. $\mathrm{L}^{-1}$ a quantidade máxima para tal elemento na água (BRASIL, 2011; WHO, 2011). Observa-se que se o valor de triagem de urânio for superado, aquele de atividade alfa total não necessariamente terá sido superado (ZORER \& ŞAHAN, 2011).

Para análise de alfa e beta total em águas, a abordagem mais comum é evaporar um volume conhecido de amostra e medir a atividade do resíduo por meio de contador proporcional ou pela técnica de espectrometria de cintilação em meio líquido (LSC, Liquid Scintillation Counting, em inglês). Esta técnica vem sendo utilizada em diversos países com o propósito de avaliar a radioatividade em águas para consumo humano (BAKIR \& BEM, 1996; RUSCONI et al., 2004; KLEINSCHMIDT, 2004; LOPES; MADRUGA; CARVALHO, 2005; SALONEN, 2006a; RUBERU; LIU; PERERA, 2008; LIN et al., 2012).

Goiás, situado no Centro-Oeste do Brasil, é o estado mais populoso da região com uma população de 6.006.788 habitantes, estimada pelo Instituto Brasileiro de Geografia e Estatística (IBGE), em 2010 (IBGE, 2010). O estado ocupa uma área de $340.111,783 \mathrm{~km}^{2}$; sua capital é Goiânia e possui, ao todo, 246 municípios. Do ponto de vista hidrográfico, Goiás dispõe de três grandes bacias brasileiras: a do rio Paraná, a do rio Tocantins e a do rio Araguaia. Seu solo é predominantemente do grupo Latossolo, quase sempre relacionado às rochas subjacentes. O relevo deste estado é de baixa declividade em sua maior parte, com modestas amplitudes altimétricas (200 a $1.200 \mathrm{~m}$ ), formado por terras planas e configurado por planaltos, chapadões, serras cristalinas e depressões intermontanas. O clima em grande parte do estado pode ser classificado como quente e subúmido, com duas estações bem definidas, um período chuvoso ( $80 \%$ das chuvas ocorrem no período de novembro a março) e outro com baixos índices pluviométricos. Sua vegetação predominante é o cerrado, embora grandes extensões de seu território se encontrem ocupadas pela monocultura e pecuária (NASCIMENTO, 1992).

As concentrações de alfa e beta total em água são frequentemente encontradas com valores menores que o limite de detecção do procedimento analítico e, consequentemente, representam um sério problema de interpretação para a análise dos dados. O limite de detecção é um limiar abaixo do qual os valores medidos não são considerados significativamente diferentes de um sinal de branco. Baixas concentrações de químicos orgânicos e inorgânicos são usualmente reportadas como "não detectadas" ou "menores que" um limiar analítico (limite de detecção). Estatísticos usam os termos "dados censurados" para um conjunto de dados em que os valores de algumas observações não são quantificados, mas sabe-se que excedem (dados censurados à direita) ou são inferiores (dados censurados à esquerda) a um valor de referência (HELSEL, 2012). Segundo Helsel (2006), a pior prática, quando se trabalha com dados censurados, é excluí-los ou eliminá-los. Isto produz uma forte tendenciosidade (viés) em todas as medidas subsequentes de localização ou testes de hipóteses. Excluir dados censurados remove a informação primária contida neles - a proporção de informações em cada grupo que se situa abaixo do limite analítico. Para aquele autor, substituir valores usando uma fração qualquer entre zero e o limite de detecção é equivalentemente arbitrário, fácil e incorreto.

A partir de uma revisão dos métodos apresentados na literatura para determinar alfa e beta totais por meio da LSC em águas, um procedimento analítico foi estabelecido visando à triagem de águas para consumo humano no estado de Goiás de modo a avaliar a sua potabilidade no quesito radioatividade. Buscou-se estabelecer um valor típico de atividade alfa e beta para águas de abastecimento público consumidas pela população goiana, por meio da aplicação de métodos estatísticos de análise de dados censurados.

\section{METODOLOGIA}

\section{Método analítico}

Foi realizado um levantamento bibliográfico dos principais fatores que afetam a determinação de alfa e beta totais em águas por LSC a fim de estabelecer um procedimento de rotina para analisar as concentrações em atividade alfa e beta totais em águas para consumo humano. Utilizaram-se espectrômetro Quantulus 1220, frasco de polietileno e coquetel de cintilação Optiphase Hisafe 3 (PerkinElmer). Para calibração, aplicaram-se soluções de $10 \mathrm{~Bq}$ de ${ }^{241} \mathrm{Am}$ e ${ }^{90} \mathrm{Sr} /{ }^{90} \mathrm{Y}$ preparadas a partir de outras certificadas fornecidas pelo Instituto de Radioproteção e Dosimetria (IRD). A fim de avaliar o desempenho do procedimento, este foi aplicado a amostras do Programa Nacional de Intercomparação para Determinação de Radionuclídeos em Amostras Ambientais, promovido pelo Instituto de Radioproteção e Dosimetria (PNI/IRD/CNEN).

\section{Coleta de águas de abastecimento público}

Os valores de radioatividade alfa e beta totais foram mensurados em águas de abastecimento público de 96 municípios do estado de Goiás, englobando todas as suas regiões ou 39\% de seus municípios. Amostras de águas para abastecimento público foram coletadas em 2010 (julho a outubro), 2011 (abril e maio), 2013 (dezembro) e 2014 (março a agosto) em estações de tratamento de água (ETA) pela Saneamento de Goiás S/A (Saneago) e pela Superintendência de Vigilância Sanitária e Ambiental (SVISA). Estas foram acidificadas com ácido nítrico em $\mathrm{pH}<2$ (APHA, 2005) para conservação e foram enviadas ao Centro Regional de Ciências Nucleares do Centro-Oeste (CRCN-CO) para avaliar alfa e beta totais. As análises foram realizadas no período de até seis meses desde a coleta, respeitando o prazo de conservação de um ano (APHA, 2005). 
A seguir, estão relacionados os municípios nos quais foram coletadas amostras de água. Está indicado, entre colchetes, o número de cada um por região e, entre parênteses, o valor de amostragem por município, totalizando 225 análises.

- Região Centro [3]: Anápolis (1), Barro Alto (1), São Luiz do Norte (2).

- Entorno do Distrito Federal [10]: Abadiânia (2), Cabeceiras (1), Cidade Ocidental (1), Corumbá de Goiás (2), Formosa (3), Luziânia (7), Mimoso de Goiás (1), Novo Gama (1), Valparaíso de Goiás (3), Vila Boa (1).

- Região Metropolitana [17]: Abadia de Goiás (3), Aparecida de Goiânia (4), Aragoiânia (2), Bela Vista de Goiás (1), Bonfinópolis (1), Caldazinha (1), Goianápolis (2), Goiânia (6), Goianira (1), Guapó (1), Hidrolândia (1), Nerópolis (2), Nova Veneza (1), Santo Antônio de Goiás (1), Senador Canedo (12), Terezópolis de Goiás (1), Trindade (2).

- Região Norte [10]: Alto Horizonte (1), Campinorte (1), Mara Rosa (2), Minaçu (1), Niquelândia (1), Nova Iguaçu de Goiás (3), Porangatu (1), Santa Terezinha de Goiás (1), São Miguel do Araguaia (2), Uruaçu (3).

- Região Nordeste [6]: Alvorada do Norte (1), Campos Belos (2), Flores de Goiás (1), Guarani de Goiás (1), Monte Alegre de Goiás (1), Sítio d’Abadia (1).

- Região Noroeste [3]: Faina (1), Goiás (3), Matrinchã (1).

- Região Oeste [22]: Adelândia (2), Americano do Brasil (1), Amorinópolis (4), Anicus (1), Aurilândia (3), Buriti de Goiás (2), Cachoeira de Goiás (2), Córrego do Ouro (2), Doverlândia (1), Firminópolis (2), Iporá (3), Israelândia (1), Ivolândia (1), Jussara (1), Mossâmedes (2), Palmeiras de Goiás (4), Palminópolis (2), Paraúna (2), Sanclerlândia (2), Santa Fé de Goiás (1), São João da Paraúna (2), São Luís de Montes Belos (4), Turvânia (2).

- Região Sul [9]: Caldas Novas (6), Itumbiara (3), Morrinhos (1), Piracanjuba (1), Porteirão (1), Professor Jamil (1), Rio Quente (1), Vicentinópolis (2).

- Região Sudeste [6]: Catalão (30), Goiandira (2), Ouvidor (1), Pires do Rio (1), São Miguel do Passa Quatro (1), Vianópolis (1).

- Região Sudoeste [9]: Acreúna (1), Aporé (1), Castelândia (1), Chapadão do Céu (6), Itajá (2), Jataí (13), Mineiros (2), Portelândia (1), Rio Verde (3).

\section{Análise estatística}

As análises estatísticas foram realizadas usando o pacote NADA para $\mathrm{R}$ (2010), que é uma coleção de rotinas e métodos para o tratamento estatístico de dados censurados. NADA para R (HELSEL, 2012) estima a média, a mediana e o desvio padrão por meio de três métodos: Kaplan-Meier (K-M), regressão em estatística de ordem (regression on order statistics - ROS) e estimativa por máxima verossimilhança (maximum likelihood estimation - MLE). Para a análise, o conjunto de dados deve incluir um vetor de valor numérico e um lógico de indicação da censura, isto é, uma lista de valores falsos ou verdadeiros que indicam se o valor numérico no conjunto de dados é censurado (true) ou não censurado (false).

\section{RESULTADOS E DISCUSSÃO}

\section{Método analítico}

Atendendo ao artigo 22 da Portaria do Ministério da Saúde 2914/2011 (BRASIL, 2011), quanto à adoção de metodologias analíticas segundo normas nacionais ou internacionais mais recentes, buscou-se implementar a norma ISO 11704:2010 de julho de 2010 (ISO, 2010). Esta apresenta um método para determinar a concentração de atividades alfa e beta totais por LSC em águas bruta e potável com sólidos totais dissolvidos abaixo de 5 g.L.-1 e quando nenhuma correção para quenching de cor for necessária. $\mathrm{O} \mathrm{pH}$ da amostra não deve ser inferior a 1,7 $\pm 0,2$ para contagem direta, ou não inferior a 2,7 $\pm 0,2$ se a pré-concentração for desejada. No procedimento de pré-concentração, uma fração de aproximadamente $200 \mathrm{~g}$ da amostra é concentrada por um fator de 10 , por evaporação em chapa aquecedora, até uma quantidade final de aproximadamente $20 \mathrm{~g}$. Uma fração da amostra concentrada é tomada para frasco de cintilação de polietileno e misturada com coquetel de cintilação, numa razão de volumes geralmente de $8 \mathrm{~mL}$ de amostra e $12 \mathrm{~mL}$ de coquetel.

Entretanto, a aplicação direta da metodologia segundo a norma ISO 11704:2010 nas análises de radioatividades alfa e beta totais para verificar o padrão de potabilidade se mostrou inviável, uma vez que uma pré-concentração é necessária para se obter os baixos limites de detecção requeridos, e as amostras são geralmente acidificadas em $\mathrm{pH}<2$ para conservação. $\mathrm{O}$ ajuste do $\mathrm{pH}$ pela adição de solução de hidróxido de amônio foi rejeitado, evitando-se o consumo de um novo reagente. Para contornar esta limitação, a amostra foi levada à secura e o resíduo foi solubilizado em meio clorídrico em concentração de 0,01 mol.L $\mathrm{L}^{-1}$, objetivando um $\mathrm{pH}>1,7 \pm 0,2$. Entretanto, a reprodutibilidade do procedimento ficou prejudicada, em função, possivelmente, da incompleta dissolução e transferência do resíduo para o frasco de cintilação. Foi então utilizado $\mathrm{HCl}$ a 0,1 mol.L $L^{-1}$. Embora a norma ISO 11704:2010 indique o uso de ácido nítrico, foi empregado o ácido clorídrico devido a este já ser utilizado pela autora em outros ensaios de discriminação alfa/beta em LSC. Além disso, Lopes, Madruga e Carvalho (2005) evitam o uso de ácido nítrico por ele reagir com o coquetel de cintilação. O efeito da utilização dos dois ácidos foi demonstrado por Eikenberg et al. (1996) e Zapata-García, Llauradó e Rauret (2012), indicando-se o emprego do meio clorídrico como mais adequado para medições de alfa e beta totais por este manter as interferências $(\tau)$ em baixos valores. Essas interferências referem-se à classificação dos pulsos alfa na janela 
de contagem beta $(\tau \alpha)$, ou vice-versa $(\tau \beta)$. No caso de se aplicar ácido nítrico, Zapata-García, Llauradó e Rauret (2012) recomendam trabalhar com o $\mathrm{pH}=2,5$. Em meio de ácido nítrico e $\mathrm{pH}<2$, a interferência alfa $(\tau \alpha)$ foi bastante afetada por pequenas variações de $\mathrm{pH}$. No intervalo de $\mathrm{pH}$ estudado no trabalho de Zapata-García, Llauradó e Rauret (2012) $(0,5-3,0)$, a interferência alfa $(\tau \alpha)$ diminuiu de 60 para $7 \%$.

A norma ISO 11704:2010 orienta quanto à escolha de reagentes e equipamentos. São recomendados tanto os coquetéis de cintilação comercialmente disponíveis adequados para discriminação alfa e beta, como aqueles à base de DIN (di-isopropil-naftaleno). Observa-se que os coquetéis mais utilizados para este fim são os da linha Ultima Gold (AB, XR e LLT) e Optiphase Hisafe (2 e 3), da PerkinElmer. A seleção do coquetel deve ser baseada em um compromisso bem balanceado entre alta capacidade de carga, resistência a quenching, alta eficiência de contagem, baixas interferências e background, principais características desejadas do coquetel de cintilação. Os trabalhos de Salonen (1993), Sanchez-Cabeza e Pujol (1995) e Fang, Jeng-Jong e Chiu (2001) apontam os coquetéis Optiphase Hisafe com menor interferência beta $(\tau \beta)$ em comparação com aqueles da família Ultima Gold. Os trabalhos de DeVol et al. (1996) e Verrezen, Loots e Hurtgen (2008) indicam o coquetel Optiphase Hisafe 3 ligeiramente menos sensível a quenching comparado aos da família Ultima Gold. Os resultados são congruentes, desde que maiores níveis de quenching ocasionam maiores interferências $(\tau)$.

Frascos de vidro de baixo conteúdo de potássio são amplamente utilizados em LSC, especialmente com solventes voláteis, e na análise de radônio. Apesar de seu baixo conteúdo de potássio, eles apresentam um maior background para medidas de ultrabaixos níveis de radiação beta devido à fluorescência do vidro, a qual contribui principalmente na região de energia do ${ }^{3} \mathrm{H}$ (KAIHOLA, 1993), e, portanto devem ser evitados. A norma ISO 11704:2010 orienta ao emprego dos frascos de cintilação de capacidade de $20 \mathrm{~mL}$ de polietileno (PE), recomendando aqueles de polietileno revestidos de Teflon' (PTFE). Sanchez-Cabeza e Pujol (1995) e Passo e Cook (1994) apontam que os frascos revestidos de Teflon melhoram a medida simultânea de alfa e beta totais, especialmente com o aumento do tempo decorrido entre o preparo da amostra e a medição, em função da não difusão do coquetel nas paredes do frasco ou da absorção de radônio do ambiente externo, fatores que podem ocorrer quando se utilizam frascos de PE. Porém, esta variação não é tão significativa para compensar o custo destes frascos, de modo que aqui optou-se em trabalhar com frascos de PE, à semelhança de Zapata-García (2011).

O tempo decorrido entre a pré-concentração e a medição é um fator que afeta os resultados de alfa e beta totais (ARNDT \& WEST, 2007; ZAPATA-GARCÍA \& LLAURADÓ, 2009; MONTAÑA et al., 2013), independentemente do método de medição adotado. Variações na atividade alfa total ocorrem, geralmente, em função da presença de
${ }^{226} \mathrm{Ra}$ e seus produtos de decaimento. O período entre a amostragem e a análise é outro fator que deve ser considerado, sobretudo para amostras com alto conteúdo de radônio (VESTERBACKA, 2005), por sua contribuição de ${ }^{210} \mathrm{~Pb}$ e ${ }^{210} \mathrm{Bi}$ na atividade beta total.

Em vista dessas considerações, estabeleceu-se a pré-concentração pela evaporação total de $100 \mathrm{~mL}$ da amostra em chapa aquecedora, temperatura baixa, evitando-se assim ebulição do líquido e superaquecimento do resíduo. Após resfriamento, o resíduo é solubilizado pela adição, em quatro etapas, de $2 \mathrm{~mL}$ de $\mathrm{HCl}$ 0,1, Bq.L-1, e a solução é transferida para um frasco de PE. Utilizam-se $12 \mathrm{~mL}$ de coquetel Optiphase Hisafe 3. A mistura é agitada e reservada em ambiente escuro e à temperatura ambiente por aproximadamente 24 horas, visando à redução da foto e quimioluminescência (TEMBA; MINGOTE; MOREIRA, 2007). Em seguida, a amostra é posicionada no equipamento para ambientação por cerca de uma hora e contada por 270 minutos, em três ciclos de repetições de 30 minutos, cada. Observa-se uma redução no desvio padrão das taxas de contagem alfa e beta quando se aplica este tempo de espera entre a adição do coquetel e a medição; em sentido contrário, nota-se uma tendência de aumento no desvio padrão das taxas de contagem quando a medição é realizada decorridos muitos dias da adição do coquetel. Zapata-García (2011) observou que, após duas semanas, a limpidez e a homogeneidade da mistura de amostra/ coquetel são comprometidas, o que pode explicar esta variância nas taxas de contagem. Medições diversas entre dois e dez dias de preparo da amostra permitem deduzir a presença ou não na amostra de um conteúdo não desprezível de ${ }^{226} \mathrm{Ra}$ (ZAPATA-GARCÍA \& LLAURADÓ, 2009; ESPASA et al., 2012).

Geralmente, as amostras de águas por nós analisadas mostram atividades abaixo da atividade mínima detectável. Assim, amostras com taxas de contagem superiores ao background têm seus espectros avaliados para detectar uma possível contribuição de ${ }^{226} \mathrm{Ra}$ e filhos, podendo uma nova alíquota ser pré-concentrada e analisada dentro de 48 horas para confirmação do resultado. Visando não prolongar o período de contagem, trabalha-se com até 12 amostras por vez, acompanhado de um branco consistindo de $100 \mathrm{~mL}$ de água deionizada acidificada em pH 2 com $\mathrm{HNO}_{3}$, uma vez que este ácido é utilizado para conservação da amostra.

\section{Espectrômetro de cintilação em meio líquido e calibração}

Para a análise de alfa e beta totais por LSC, é necessário um espectrômetro de cintilação com opção de discriminação alfa e beta, preferencialmente um equipamento de baixos níveis de background de modo a se obter baixos limites de detecção. O espectrômetro Quantulus 1220 (PerkinElmer) tem sido o mais utilizado para este fim (SANCHEZ-CABEZA \& PUJOL, 1995; RUSCONI et al., 2004; VESTERBACKA, 2005; SALONEN, 2006a; RUBERU; LIU; PERERA, 
2008; LIN et al., 2012; ZAPATA-GARCÍA; LLAURADÓ; RAURET, 2012; MONTAÑA et al., 2013). Os analisadores da linha Tri-Carb (PerkinElmer) são também bastante empregados, tal como o modelo equipado com detector de guarda Bismuth Germanium Oxide (BGO) designado para contagens das amostras de atividades extremamente baixas (KLEINSCHMIDT, 2004; LOPES; MADRUGA; CARVALHO, 2005), além de outros modelos que dispõem da função de discriminação alfa e beta (BAKIR; BEM, 1996; WONG; SOLIMAN; PERERA, 2005; RUBERU; LIU; PERERA, 2008; DIAS et al., 2009). O contador LSC portátil Triathler da HIDEX Oy é outro equipamento que tem sido aplicado na determinação de alfa e beta totais em águas (STAFFOVÁ; NĚMEC; JOHN, 2006; WISSER; FRENZEL; DITTMER, 2006).

Para a determinação simultânea das emissões alfa e beta, deve-se estabelecer o valor ótimo do parâmetro da discriminação de pulsos (PSD) a fim de separar corretamente os pulsos alfas dos betas, mantendo-se as interferências $\tau \alpha$ e $\tau \beta$ nos valores mínimos possíveis. A abordagem geralmente adotada para o PSD ótimo é igualar os valores de interferências $\tau \alpha$ e $\tau \beta$. A calibração deve ser feita por meio de radionuclídeos de energia próxima à média observada nas amostras reais. As janelas de contagem devem ser escolhidas de modo a incluir todos os emissores alfa e beta de energia $>0,2 \mathrm{MeV}$ para que os resultados sejam comparáveis com outras técnicas de medição (ISO, 2010). Não só os radionuclídeos empregados na calibração, como as janelas de contagem, podem afetar o valor ótimo de discriminação alfa/beta (ZAPATA-GARCÍA \& LLAURADÓ, 2009). Assim, as janelas de contagem foram definidas de modo a contemplar uma ampla faixa de emissores alfa, 400 a 900 canais, enquanto a contribuição dos emissores beta de baixa energia como ${ }^{228} \mathrm{Ra}\left(\mathrm{E}_{\max }=0,039 \mathrm{MeV}\right) \mathrm{e}^{210} \mathrm{~Pb}$ $\left(\mathrm{E}_{\max }=0,064 \mathrm{MeV}\right)$ foi excluída da janela de contagem beta definida em 400 a 1.000 canais.

${ }^{236} \mathrm{U}(4,494 \mathrm{MeV})$ é uma escolha conveniente quando se analisa a radioatividade em águas naturais em função de sua energia ser mais próxima dos radionuclídeos emissores alfa mais prováveis de serem encontrados: ${ }^{238} \mathrm{U}(4,196 \mathrm{MeV} ; 79,0 \%),{ }^{234} \mathrm{U}(4,775 \mathrm{MeV} ; 71,4 \%)$ e ${ }^{226} \mathrm{Ra}$ (4,784 MeV; 94,5\%). Embora ${ }^{241} \mathrm{Am}$ (5,485 MeV; 84,6\%) seja comumente utilizado como referência de emissor alfa nas determinações de alfa total, este é mais indicado para verificar a contaminação por radionuclídeos artificiais. Observa-se que a dependência da eficiência de detecção alfa da energia do emissor alfa é pequena, mas a interferência alfa $(\tau \alpha)$ é superior para as partículas alfa de menor energia (SALONEN, 2006b), de modo que a calibração usando ${ }^{241} \mathrm{Am}$ pode conduzir a erros sistemáticos na determinação da atividade alfa total. Zapata-García, Llauradó e Rauret (2012) observaram que a calibração com emissor alfa de mais baixa energia se aplica para uma amostra com emissor de mais alta energia, porém o inverso pode levar a erros devido ao aumento da interferência alfa.
Uma mistura de ${ }^{90} \mathrm{Sr}\left(\mathrm{E}_{\max }=0,546 \mathrm{MeV}\right) \mathrm{e}^{90} \mathrm{Y}\left(\mathrm{E}_{\max }=2,284 \mathrm{MeV}\right)$ é comumente utilizada como referência para atividade beta total. ${ }^{40} \mathrm{~K}$ $\left(\mathrm{E}_{\max }=1,312 \mathrm{MeV}\right)$, o emissor beta mais provável em águas naturais, possui energia de emissão beta entre ${ }^{90} \mathrm{Sr} \mathrm{e}{ }^{90} \mathrm{Y}$. Enquanto a eficiência de detecção beta é altamente dependente da energia do emissor, a otimização do PSD não é afetada, conforme observado por Zapata-García, Llauradó e Rauret (2012). Espasa et al. (2012) destacam que o elemento crítico para se obter resultados satisfatórios pela técnica LSC é a otimização das janelas de contagem e do PSD por meio de padrões adequados. As condições de trabalho devem ser escolhidas de modo que as interferências sejam baixas $(<5 \%)$. Nessa situação, as incertezas nas atividades são geralmente incluídas no intervalo de valores definidos pela incerteza total. De outro modo, é necessário considerar as interferências nos cálculos de atividade (ISO, 2010); contudo, aplicar correções de interferência alfa e beta é indicado somente se o radionuclídeo ensaiado for o mesmo utilizado na calibração, similarmente à técnica de contador proporcional (SEMKOW \& PAREKH, 2001).

Neste estudo, a calibração foi realizada em um espectrômetro Quantulus utilizando soluções de ${ }^{241} \mathrm{Am} \mathrm{e}{ }^{90} \mathrm{Sr} /{ }^{90} \mathrm{Y}$ de $10 \mathrm{~Bq}$ de atividade, preparadas a partir de soluções certificadas, por meio do mesmo procedimento de evaporação total adotado para as amostras. Em meados de 2013, observou-se uma redução do valor de background alfa e beta supostamente devido a novo lote de reagentes. Os principais parâmetros de calibração estão resumidos na Tabela 1. Verificou-se que a redução da janela de contagem para emissores beta de média à alta energia levou a uma baixa eficiência beta.

Apesar das limitações quanto à utilização do ${ }^{241} \mathrm{Am}$ para avaliar a radioatividade natural, este nuclídeo foi utilizado por estar disponível no momento desta pesquisa. A Figura 1 mostra as interferências alfa $(\tau \alpha)$, beta $(\tau \beta)$ e total $(\tau)$. PSA (Pulse Shape Analyser) é o PSD

Tabela 1 - Parâmetros de determinação das atividades alfa e beta total por espectrometria de cintilação em meio líquido.

\begin{tabular}{|c|c|}
\hline Parâmetro & Valor $^{\mathrm{a}}$ \\
\hline Padrão de calibração & Alfa $={ }^{241} \mathrm{Am}, \mathrm{Beta}={ }^{90 \mathrm{Sr} / 90} \mathrm{Y}$ \\
\hline Janelas de contagem & Alfa=400 a 900; Beta=400 a 1.000 \\
\hline Bias & Baixo (Low) \\
\hline Interferência (\%) & $\tau \alpha=1,7 \pm 0,2 ; \tau \beta=2,5 \pm 0,3 ;$ Total, $\tau=4,2 \pm 0,5$ \\
\hline Eficiência (\%) & $\varepsilon \alpha=99,9 \pm 1,1 ; \varepsilon \beta=70,0 \pm 0,6$ \\
\hline Quenching & $S Q P(E)=714 \pm 4$ \\
\hline Discriminação alfa/beta & $P S A=80$ \\
\hline Background (cpm) & Bkg $\alpha=0,44 \pm 0,16 ; \mathrm{Bkg} \beta=1,60 \pm 0,26$ \\
\hline Tempo de contagem & $\begin{array}{l}270 \text { minutos: três ciclos de três } \\
\text { repetições de } 30 \text { minutos }\end{array}$ \\
\hline Limite de detecção (Bq) & Alfa $=0,002 ;$ Beta $=0,006$ \\
\hline
\end{tabular}

${ }^{a}$ As incertezas correspondem a um desvio padrão de três replicatas de branco e padrões contados em dois períodos de intervalo de sete dias. Valores de background obtidos em 2014. PSA: pulse shape analyser 
utilizado pelo equipamento Quantulus. O PSA 80 foi escolhido pela minimização da interferência total $(\tau)$ e não aquele pela minimização de ambas interferências, PSA 85. Observa-se que, deste modo, o PSA foi deslocado para um valor inferior, estando mais adequado à avaliação da radioatividade natural.

Os espectros alfa e beta dos padrões de ${ }^{241} \mathrm{Am} \mathrm{e}{ }^{90} \mathrm{Sr} /{ }^{90} \mathrm{Y}$ estão mostrados nas Figuras 2 e 3, respectivamente. Foi considerado um espectro de background consistindo de $8 \mathrm{~mL}$ de $\mathrm{HCl}$ 0,1 mol.L-1. Para o cálculo das eficiências, interferências, concentração em atividade e atividade mínima detectável (AMD), foram aplicadas as fórmulas da norma ISO 11704:2010. As interferências $\tau \alpha$ e $\tau \beta$ não foram consideradas no cálculo das atividades por estarem abaixo de $5 \%$.

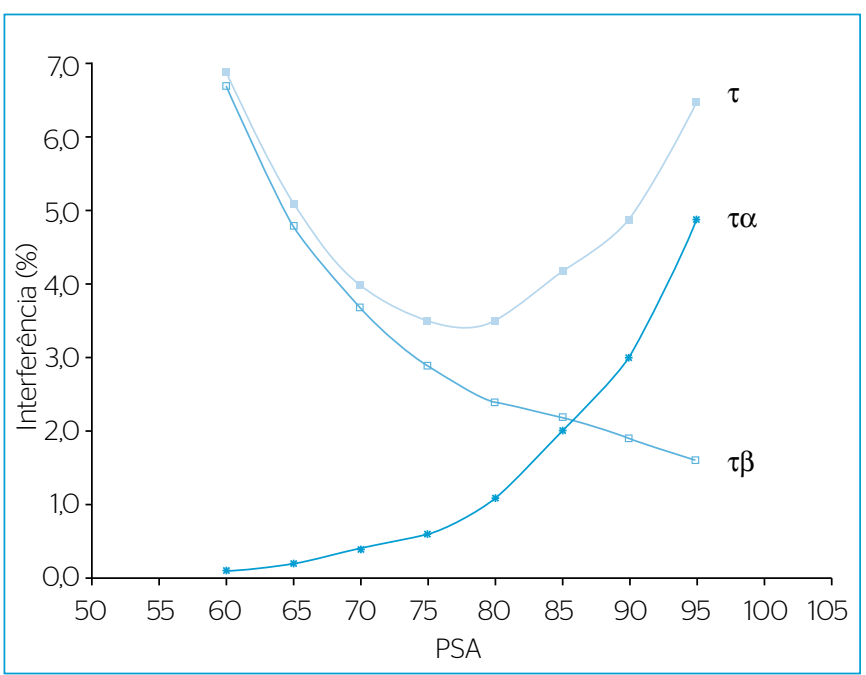

Figura 1 - Interferências alfa $(\tau \alpha)$, beta $(\tau \beta)$ e total $(\tau)$ em função do valor de pulse shape analyser (PSA) para ${ }^{241} \mathrm{Am}$ e ${ }^{90} \mathrm{Sr} /{ }^{90} \mathrm{Y}$ em HCl 0,1 mol..-1 e coquetel Optiphase Hisafe 3 na razão 8:12 mL, frasco de polietileno.

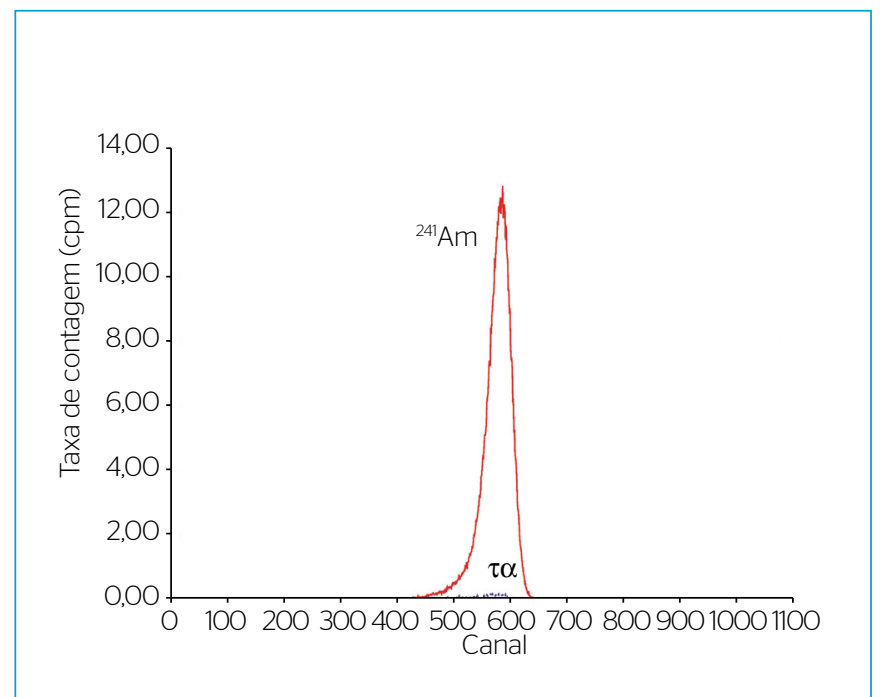

Figura 2 - Espectros alfa e beta em pulse shape analyser (PSA) 80 para padrão de ${ }^{241} \mathrm{Am}$ em $\mathrm{HCl} 0,1 \mathrm{~mol}^{-1} \mathrm{~L}^{-1}$ e coquetel Optiphase Hisafe 3 na razão 8:12 mL, frasco de polietileno.

\section{Avaliação de desempenho do método}

O desempenho do método foi avaliado por meio da análise de alfa e beta totais em amostras de água do PNI/IRD/CNEN. Este ocorre regularmente desde 1991 e é de suma importância para o Brasil, visto ser o único programa de intercomparação no país na área dos ensaios de radionuclídeos em amostras ambientais. São realizadas três rodadas anuais em abril, agosto e dezembro. Para maior facilidade de transporte, a amostra é enviada concentrada em $\mathrm{HNO}_{3}$ $1 \%$, devendo ser diluída de acordo com os procedimentos indicados. Os resultados são, então, relatados para as amostras diluídas. Deverão ser analisadas no mínimo três alíquotas de cada amostra para que seja possível a avaliação estatística dos resultados. O programa trabalha com vários radionuclídeos naturais e artificiais de interesse em amostras ambientais. Os ensaios de alfa e beta totais têm um grande número de participações, mas exibem pior desempenho, apesar de sua relativa simplicidade ao ser comparado com a análise de radionuclídeos específicos, que apresentam melhor desempenho (TAUHATA et al., 2006). Esta mesma tendência foi observada em um exercício de intercomparação entre laboratórios espanhóis, cujo objetivo foi determinar as concentrações em atividade alfa total e para o isótopo ${ }^{234} \mathrm{U}$, existente em uma amostra de água subterrânea natural (ESPASA et al., 2012).

O desempenho dos resultados de alfa total foi satisfatório, com baixos coeficientes de variação, conforme mostrado na Tabela 2. Entretanto, aquele de beta total da amostra da rodada de agosto de 2011 (Tabela 3) foi subestimado, ainda que dentro dos limites de advertência. A Figura 4 indica o espectro LSC desta amostra. A característica do alfa sugere a presença de ${ }^{241} \mathrm{Am}$, enquanto o beta sugere a presença de ${ }^{137} \mathrm{Cs}\left(\mathrm{E}_{\max }=0,514 \mathrm{MeV} ; \mathrm{I}=94,4 \%\right) ; \mathrm{E}_{\max }=1,176 \mathrm{MeV}$;



Figura 3 - Espectros alfa e beta em pulse shape analyser (PSA) 80 para padrão de ${ }^{90} \mathrm{Sr} /{ }^{\circ} \mathrm{Y}$ em HCl 0,1 mol.L-1 e coquetel Optiphase Hisafe 3 na razão 8:12 mL, frasco de polietileno. 
$\mathrm{I}=5,6 \%) . \mathrm{O}{ }^{137} \mathrm{Cs}$ decai em ${ }^{137 \mathrm{~m}} \mathrm{Ba}$, estando em equilíbrio com este, o qual decai em $85,1 \%$ por raios gama $(\mathrm{E}=0,661 \mathrm{MeV})$ e $9,4 \%$ por elétrons de conversão. Raios gama e elétrons de conversão podem ser detectados por LSC, sendo registrados no canal beta. Neste caso, o padrão de calibração utilizado $\left({ }^{90} \mathrm{Sr} /{ }^{90} \mathrm{Y}\right)$ não é indicado para este radionuclídeo, assim como a janela de contagem previamente estabelecida. As atividades foram recalculadas considerando-se a janela de contagem beta ( 1 a 1.000 canais), com eficiência de detecção $\varepsilon \beta=90 \%$, obtendo-se um desempenho satisfatório, como observado nos resultados das demais rodadas (Tabela 3 ).

Zapata-Garcia et al. (2012) reportaram bons resultados na validação do seu método usando amostras sintéticas, mas verificaram que adequações deviam ser realizadas, pois diferenças foram observadas nas amostras reais. Uma vez que as características dos radionuclídeos utilizados no PNI/IRD/CNEN são diferentes daquelas das amostras de águas dos sistemas de ETA, um programa de qualidade interna está em implementação para acreditação do método em uma matriz similar às amostras reais.

\section{Atividades alfa e beta totais em águas de Goiás}

Amostras de água tratada destinadas para consumo humano foram analisadas para concentrações de atividade alfa e beta totais por LSC, usando as calibrações apresentadas na Tabela 1. Para as coletas de 2010 e 2011, foram utilizadas alíquotas de 250 e $100 \mathrm{~mL}$ respectivamente, atendendo igualmente ao limiar de atividade. Em 2013 e 2014, manteve-se a alíquota de $100 \mathrm{~mL}$, contudo notou-se uma redução nos valores de background alfa e beta. Isso gerou três diferentes concentrações mínimas detectáveis (Bq. $\mathrm{L}^{-1}$ ) para cada atividade alfa e beta, justificando novamente a utilização de métodos estatísticos aos dados (multi) censurados para o estabelecimento dos valores de localização (mediana e média).

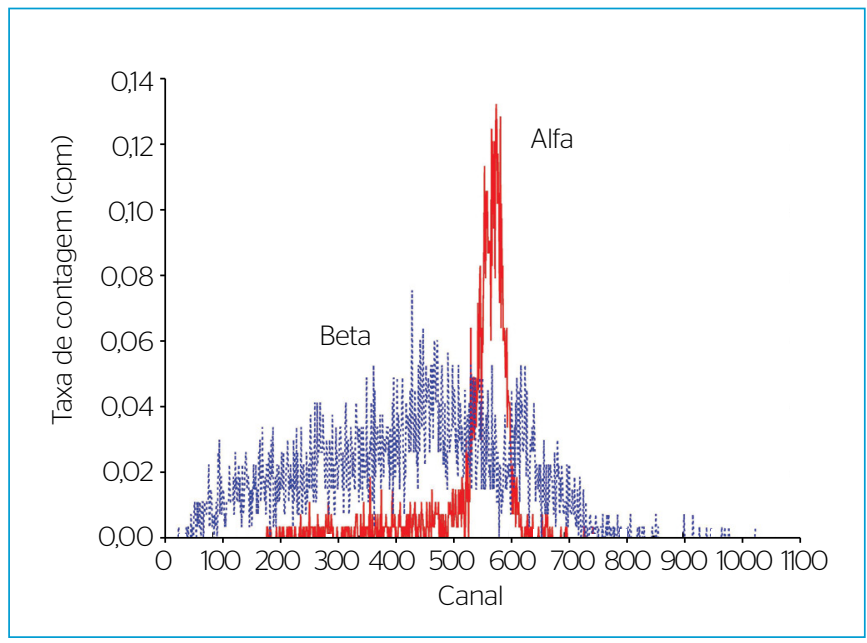

Figura 4-Espectros alfa e beta por espectrometria de cintilação em meio líquido de amostra de água do Programa Nacional de Intercomparação para Determinação de Radionuclídeos em Amostras Ambientais, PNI/ IRD/CNEN para análise de alfa e beta total, rodada de agosto/2011.

Tabela 2 - Atividade alfa total (Bq.L-1) por espectrometria de cintilação em meio líquido e desempenho analítico no Programa Nacional de Intercomparação para Determinação de Radionuclídeos em Amostras Ambientais.

\begin{tabular}{|c|c|c|c|c|}
\hline \multirow{2}{*}{ Rodada } & \multicolumn{4}{|c|}{ Alfa total } \\
\hline & Referência ${ }^{\mathrm{a}}$, U & Experimental $^{\mathrm{a}}, \mathrm{X}$ & $\mathrm{D}^{\mathrm{b}}$ & $\mathrm{CV}^{\mathrm{c}}$ \\
\hline Agosto de 2011 & $0,860 \pm 0,170$ & $0,902 \pm 0,036$ & 0,43 & 4 \\
\hline Dezembro de 2011 & $1,250 \pm 0,250$ & $1,246 \pm 0,019$ & $-0,03$ & 2 \\
\hline Abril de 2012 & $0,520 \pm 0,100$ & $0,526 \pm 0,001$ & 0,11 & $\mathrm{O}$ \\
\hline Agosto de 2012 & $1,050 \pm 0,210$ & $1,054 \pm 0,035$ & 0,03 & 3 \\
\hline
\end{tabular}

${ }^{a}$ As incertezas correspondem a um desvio padrão. ${ }^{\text {b: }} \mathrm{D}=$ desvio normalizado, utilizado como parâmetro de avaliação e calculado pela expressão $D=\frac{X-U}{S{ }^{\prime}}$. c: $C V=$ coeficiente de variação, definido por $C V=\frac{s}{x} \cdot 100 \%$, onde su e s são o desvio padrão dos valores de referência e experimental, respectivamente.

Tabela 3 - Atividade beta total (Bq.L-1) por espectrometria de cintilação em meio líquido e desempenho analítico no Programa Nacional de Intercomparação para Determinação de Radionuclídeos em Amostras Ambientais.

\begin{tabular}{l|c|c|c|c}
\multirow{2}{*}{ Rodada } & \multicolumn{4}{|c}{ Beta total } \\
\cline { 2 - 5 } Agosto de 2011 & Referência, $\mathrm{U}$ & Experimental, $\mathrm{X}$ & $\mathrm{D}^{\mathrm{c}}$ & $\mathrm{CV}^{\mathrm{d}}$ \\
\hline Dezembro de 2011 & $1,380 \pm 0,280$ & $1,006 \pm 0,104^{\mathrm{a}}$ & $-2,31$ & 7 \\
\hline Abril de 2012 & $2,210 \pm 0,440$ & $1,235 \pm 0,085^{\mathrm{b}}$ & $-0,89$ & 6 \\
\hline Agosto de 2012 & $0,960 \pm 0,190$ & $2,145 \pm 0,139^{\mathrm{b}}$ & $-0,26$ & 2 \\
\hline
\end{tabular}

a.b Janela de contagem beta: (a) 400 - 1.000; (b) 1 - 1.000. As incertezas correspondem a um desvio padrão. ' D = desvio normalizado, utilizado como parâmetro de avaliação e calculado pela expressão $D=\frac{X-U}{S U / \sqrt{3}}$ : $C V=$ coeficiente de variação, definido por $C V=\frac{S}{X} \cdot 100 \%$, onde su e $s$ são o desvio padrão dos valores de referência e experimental,
respectivamente. 
O resumo dos dados de atividades alfa e beta totais em águas de Goiás está apresentado na Tabela 4. Muitas amostras indicaram valores abaixo da concentração mínima detectável. Observa-se que 44,4 e $55,1 \%$ dos dados são censurados para atividades alfa e beta, respectivamente. A maioria das amostras $(n=224,99,6 \%)$ mostrou concentrações de atividades abaixo do valor de referência da portaria do Ministério da Saúde 2914/2011, isto é, abaixo de 0,5 e 1,0 Bq.L-1 para alfa e beta totais, respectivamente. Uma amostra de 2011 apresentou valor de alfa total $\left(0,7 \pm 0,1 \mathrm{~Bq} \cdot \mathrm{L}^{-1}\right)$ acima daquele de referência para atividade alfa. Esta amostra também apresentou o valor máximo de atividade beta de $0,6 \pm 0,2 \mathrm{~Bq} . \mathrm{L}^{-1}$, que está dentro do limite de potabilidade para atividade beta. Em nova campanha de amostragem em 2014, este ponto de amostragem indicou atividade alfa de $0,20 \pm 0,06 \mathrm{~Bq} \cdot \mathrm{L}^{-1}$ e beta de $0,34 \pm 0,08$ Bq. $\mathrm{L}^{-1}$. A característica do espectro alfa de LSC (Figura 5) desta amostra sugere a presença de ${ }^{226} \mathrm{Ra}$ ou urânio natural. Recomenda-se que a amostra seja analisada para urânio e ${ }^{226} \mathrm{Ra}$, e que este ponto de amostragem seja monitorado em maior frequência para um resultado conclusivo.

A mediana foi utilizada como valor típico para alfa e beta totais por ser mais apropriada, como na maioria dos dados ambientais frequentemente modelados usando uma distribuição log-normal (Tabela 5). O método padrão para estimar o resumo estatístico dos dados de sobrevivência censurados é o não paramétrico de Kaplan-Meier. Este foi adotado para os dados de estimação do alfa total, uma vez que é recomendado para dados multicensurados com até $50 \%$ de censura. Já para os dados de beta total, aplicou-se o método MLE devido aos valores serem censurados em mais de 50\%.

As atividades típicas estimadas são muito baixas, 0,030 e 0,054 Bq.L.-1 para alfa e beta totais, respectivamente. Estes valores indicam ainda que as águas de abastecimento público monitoradas atendem ao critério de potabilidade da portaria do Ministério da Saúde no quesito radioatividade. Considerando a grande percentagem de dados censurados, estes valores podem ser reduzidos caso novas análises com limites de detecção ainda inferiores sejam realizadas.

Para uma caracterização radiológica das águas de abastecimento público em Goiás, sugere-se a determinação dos contribuintes emissores alfa, em especial urânio e ${ }^{226} \mathrm{Ra}$. Confrontando-se valores obtidos de alfa total com os teores de ${ }^{226} \mathrm{Ra}$ em águas subterrâneas nas cercanias de Goiânia, <0,004-0,023 Bq.L-1 (LAURIA et al., 2004), observou-se que o limite superior de rádio é inferior à concentração típica para alfa total. Para beta total, o valor típico foi comparado com a contribuição do ${ }^{40} \mathrm{~K}$ estimada a partir dos teores de potássio em águas de ETA da região metropolitana de Goiânia, com 0,8 a 2,3 mg.L.-1 (COSTA, 2010). A atividade específica do ${ }^{40} \mathrm{~K}$ é $31,3 \mathrm{~Bq} \cdot \mathrm{g}^{-1}$ de potássio, o que corresponde a $27,9 \mathrm{~Bq} \cdot \mathrm{g}^{-1}$ de atividade beta. Assim, o valor típico de beta total

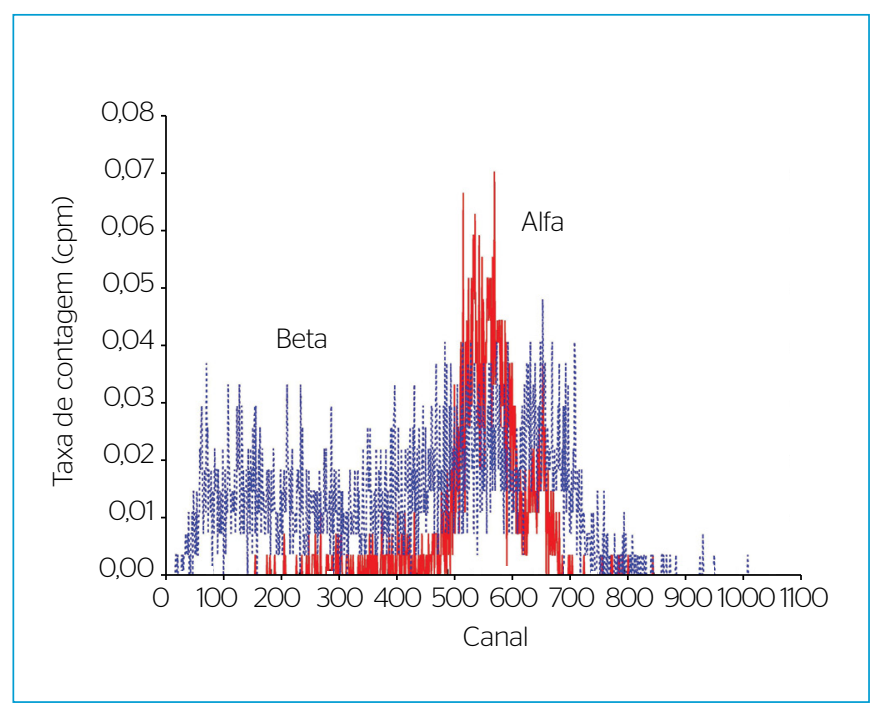

Figura 5 - Espectros alfa e beta por espectrometria de cintilação em meio líquido de amostra de água.

Tabela 4 - Resumo dos dados das atividades alfa e beta total $\left(B q \mathrm{~L}^{-1}\right)$ em águas de Goiás.

\begin{tabular}{l|c|c|c|c|c} 
Atividade & Número de dados & $\begin{array}{c}\text { Número de dados } \\
\text { censurados }\end{array}$ & $\begin{array}{c}\text { Percentagem de dados } \\
\text { censurados }\end{array}$ & Valor mínimo & \multicolumn{1}{c}{ Valor máximo } \\
\hline Alfa & 225 & 100 & 44,4 & 0,01 & 0,7 \\
\hline Beta & 225 & 124 & 55,1 & 0,04 & 0,6 \\
\hline
\end{tabular}

Tabela 5 - Resumo estatístico das atividades alfa e beta total (Bq.L-1) em águas de Goiás, usando três métodos de estimação.

\begin{tabular}{l|c|c|c|c}
\hline \multirow{4}{*}{ Atividade (pct.cen)a } & Método de estimação & Mediana & Média & Desvio padrão \\
\cline { 2 - 5 } & Kaplan-Meier & 0,030 & 0,051 & 0,068 \\
\cline { 2 - 5 } & ROS & 0,030 & 0,053 & 0,068 \\
\cline { 2 - 5 } & MLE & 0,020 & 0,066 & 0,205 \\
\cline { 2 - 5 } Beta (55,\%) & Kaplan-Meier & 0,040 & 0,092 & 0,086 \\
\cline { 2 - 5 } & ROS & 0,059 & 0,089 & 0,089 \\
\hline
\end{tabular}

aNúmero de dados = 225; pct.cen: percentagem de dados censurados. ROS: regressão em estatística de ordem; MLE: estimativa por máxima verossimilhança. 
estabelecido neste estudo encontra-se dentro da faixa de atividade beta em função de ${ }^{40} \mathrm{~K}\left(0,02\right.$ a 0,06 Bq.L $\left.\mathrm{L}^{-1}\right)$.

\section{CONCLUSÃO}

De acordo com os valores de atividades típicas estimados neste estudo de $0,030 \mathrm{~Bq} \cdot \mathrm{L}^{-1}$ para alfa total e $0,054 \mathrm{~Bq} \cdot \mathrm{L}^{-1}$ para beta total, nas águas para abastecimento público do estado de Goiás, verifica-se que estas encontram-se dentro dos padrões de potabilidade de água quanto ao quesito radioatividade. O método LSC se mostrou adequado para a mensuração das atividades alfa e beta totais em águas naturais e para consumo humano, e ajustes na metodologia de análise asseguram resultados de boa exatidão e precisão. Dentre os fatores que influenciam a exatidão e a precisão dos resultados analíticos, destacam-se: pré-concentração térmica, tipo de ácido, janela de contagem e padrão de calibração.

\section{REFERÊNCIAS}

AMERICAN PUBLIC HEALTH ASSOCIATION - APHA. (2005) Standard methods for the examination of water and wastewater. $21^{\text {st }}$ ed. Washington, DC: APHA, AWWA, WEF. 1082p.

ARNDT, M.F. \& WEST, L.E. (2007) An experimental analysis of some of the factors affecting gross alpha-particle activity with an emphasis on ${ }^{226}$ Ra and its progeny. Health Physics, v. 92, p. 148-156.

BAKIR, Y.Y.\&BEM, H. (1996) Application of pulse decay discrimination liquid scintillation counting for routine monitoring of radioactivity in drinking water. In: COOK, G.T.; HADKNESS, D.D.; MACKENZIE, A.B.; MILLER, B.F.; SCOTT, E.M. (Eds.). Liquid scintillation spectrometry 1994. [S.I.]: Radiocarbon, p. 293-299.

BRASIL. (2011) Portaria MS no 2914, de 12 de dezembro de 2011. Diário Oficial da União, Brasília, DF, Seção 1, p. 39-46.

COSTA, J.S. (2010) Análise química da água nas estações de tratamento de água (ETAs) da região metropolitana de Goiânia-GO e sua relação com a saúde. Dissertação (Mestrado) - Pontifícia Universidade Católica de Goiás, Goiás, Brasil.

DEVOL, T.A.; BROWN, D.D.; LEYBA, J.D.; FJELD, R.A. (1996) A comparison for four aqueous-miscible liquid scintillation cocktails with an alpha/beta discriminating Wallac 1415 liquid scintillation counter. Health Physics. v. 70, p. 41-46.

DIAS, F.F.; TADDEI, M.H.T.; PONTEDEIRO, E.M.B.D.; JACOMINO, V.F. (2009) 2009 International Nuclear Atlantic Conference - INAC 2009. Anais... Rio de Janeiro: INAC.

EIKENBERG, J.; FIECHTNER, A.; RUETHI, M.; ZUMSTEG, I. (1996) A Rapid Screening Method for Determining Gross Alpha Activity in Urine Using Alpha/Beta LSC. In: COOK, G.T; HADKNESS, D.D.; MACKENZIE, A.B.; MILLER, B.F.; SCOTT, E.M. (Eds.). Liquid Scintillation Spectrometry 1994. [S.I.]: Radiocarbon, p. 283-292.

ESPASA, A.B.; MERINO, J.A.C.; TARRAGÓ, M.L.; MURCIANO, I.V. (2O12) Dificuldades existentes en la medida de la actividad alfa total en muestras de água. Alfa, n. 18, p. 56-62.

FANG, T.W.S.F.; JENG-JONG W.; CHIU, J.H. (2001) Comparison of alpha/ beta separation performance of commercially available scintillation cocktails counting by Quantulus 1220 liquid scintillation counter. Anais... Honolulu: Radiochemical Measurement Conference.
HELSEL, D.R. (2012) Statistics for censored environmental data using Minitab and R. $2^{\text {nd }}$ ed. New York: John Wiley and Sons.

HELSEL, D.R. (2006) Fabricating data: how substituting values for nondetects can ruin results, and what can be done about it Chemosphere, v. 65, p. 2434-2439.

INSTITUTO BRASILEIRO DE GEOGRAFIA E ESTATÍSTICA - IBGE. (2010) Censo 2010. Disponível em: <http://www.censo2010. ibge.gov.br/dados_divulgados/index. php?uf=52>. Acesso em: 10 out. 2011.

INTERNATIONAL ORGANIZATION FOR STANDARDIZATION - ISO (2010) ISO 11704:2010 - Water quality - Measurement of gross alpha and beta activity concentration in non-saline water - Liquid scintillation counting method. Geneva, Switzerland.

KAlHOLA, L. (1993) Glass vial background reduction in liquid scintillation counting. Science of the Total Environment, v. 130-131, p. 297-304.

KLEINSCHMIDT, R.I. (2004) Gross alpha and beta activity analysis in water - a routine laboratory method using liquid scintillation analysis. Applied Radiation and Isotopes, v. 61, n. 2-3, p. 333-338.

LAURIA, D.C.; SILVA-JÚNIOR, M.M.; NICOLI, I.G.; DA COSTA, H.F.; PIMENTA, L.R.; DE CARVALHO, L.L.; CORREA, R.S. (2004). Anais... Cuiabá: XII Congresso Brasileiro de Águas Subterrâneas.

LIN, C.F.; WANG, J.J.; HUANG, J.C.; YEH, C.H.; YUAN, M.C.; CHANG, B.J. (2012) Evaluating practicability of a LSC method for routine monitoring gross alpha and beta activities in water samples in Taiwan. Applied Radiation and Isotopes, v. 70, p. 1981-1984.

LOPES, I.; MADRUGA, M.J.; CARVALHO, F.P. (2005) Application of liquid scintillation counting techniques to gross alpha, gross beta, radon and radium measurement in Portuguese waters. In: NORM IV - Naturally occurring radioactive materials. p. 357-367.

MONTAÑA, M.; FONS, J.; CORBACHO, J.; CAMACHO, A.; ZAPATA-GARCIIA, D.; GUILLÉN, J.; SERRANO, I.; TENT, J.; BAEZA, A.; LLAURADÓ, M.; VALLÉS, I.J. (2013) A comparative experimental study of gross alpha methods in natural waters. Journal of Environmental Radioactivity, v. 118, p. 1-8. 
NASCIMENTO, M.A.L. (1992) Geomorfologia do Estado de Goiás. Boletim Goiano de Geografia, v. 12, n. 1, p. 1-22.

PASSO JR, C.J.; COOK, G.T. (1994) Handbook of environmental liquid scintillation spectrometry: a compilation of theory and methods. Meriden: Packard Instrument Company.

R DEVELOPMENT CORE TEAM. (2010) R: A Language and Environment for Statistical Computing. Vienna, Austria: R Foundation for Statistical Computing.

RUBERU, S.R.; LIU, Y.G.; PERERA, S.K. (2008) An improved liquid scintillation counting method for the determination of gross alpha activity in groundwater wells. Health Physics, v. 95, n. 4, p. 397-406.

RUSCONI, R:; AZZELLINO, A.; BELLINZONA, S.; FORTE, M.; GALLINI, R.; SGORBATI, G. (2004) Assessment of drinking water radioactivity content by liquid scintillation counting: set up of high sensitivity and emergency procedures. Analytical and Bioanalytical Chemistry, v. 379, p. 247-253.

SALONEN, L. (1993) Measurements of low levels of ${ }^{222} \mathrm{Rn}$ in water with different commercial liquid scintillation counters and pulse-shape analysis. In: NOAKES, J.E.; SSHÖNHOFER, F.; POLACH, H.A. (Eds). Liquid Scintillation Spectrometry 1992. [S.I.]: Radiocarbon, p. 361-372.

SALONEN, L. (2006a) Alpha/beta liquid scintillation spectrometry in surveying Finnish groundwater samples. Radiochemistry, v. 48, p. 606-612.

SALONEN, L. (2006b). Alpha spillover depends on alpha energy: a new finding in alpha/beta liquid scintillation spectrometry. In: CHALUPNIK, S.; SCHÖNHOFER, F.; NOAKES, J. (Eds.). LSC 2005 Advances in Liquid Scintillation Spectrometry. [S.I.]: Radiocarbon, p. 135-148.

SANCHEZ-CABEZA, J.A. \& PUJOL, L.I. (1995) A rapid method for the simultaneous determination of gross alpha and beta activities in water samples using a low background liquid scintillation counter. Health Physics, v. 68, p. 674-682.

SEMKOW, T.M. \& PAREKH, P.P. (2001) Principles of gross alpha and beta radioactivity detection in water. Health Physics, v. 81, p. 567-574.

STAFFOVA, P.; NËMEC, M.; JOHN, J. (2006) Determination of gross alpha and beta activities in water samples by liquid scintillation counting. Czechoslovak. Journal of Physics, v. 56, p. D299-D305.
TAUHATA, L.; VIANNA, M.E.C.M.; DE OLIVEIRA, A.E.; FERREIRA, A.C.; BRAGANÇA, M.J.C.; CLAIN, A.F.; DE FARIA, R.Q. (2006) The Brazilian national intercomparison program (PNI/IRD/ CNEN): evaluation of 15 years of data. Journal of Environment Radioactivity, v. 86, p. 384-390.

TEMBA, E.S.C.; MINGOTE, R.M.; MOREIRA, R.M. (2007) Tritium determination in aqueous samples by using LSC Quantulus in CDTN, Brazil. In: 2007 International Nuclear Atlantic conference INAC 2007, Santos, Brazil, CD-ROM.

VERREZEN, F.; LOOTS, H.; HURTGEN, C. (2008) A performance comparison of nine selected liquid scintillation cocktails. Applied and Radiation and Isotopy, v. 66, p. 1038-1042.

VESTERBACKA, P. (2005) ${ }^{238} \mathrm{U}$ series radionuclides in Finnish groundwater-based drinking water and effective doses. Tese de Doutorado - Universidade de Helsinki, Finlândia.

WORLD HEALTH ORGANIZATION - WHO. (2011) Guidelines for drinking-water quality. Genebra, Suiça: World Health Organization.

WISSER, S.; FRENZEL, E.; DITTMER, M. (2006) Innovative procedure for the determination of gross-alpha/gross-beta activities in drinking water. Applied and Radiation Isotopy, v. 64, p. 368-372.

WONG, C.T; SOLIMAN, V.M.; PERERA, S.K. (2005) Gross alpha/ beta analyses in water by liquid scintillation counting. Journal of Radioanalytical and Nuclear Chemistry, v. 264, p. 357-363.

ZAPATA-GARCIA, D. (2011) Determinació d'índexs d'activitat alfa total i beta total per escinti-lació líquida. Tese de Doutorado Universidade de Barcelona, Espanha.

ZAPATA-GARCIA, D. \& LLAURADÓ, M. (2009) Study of Instrumental Parameters Affecting the Simultaneous Measurement of Gross Alpha and Gross Beta Activities in Water Samples. In: EIKENBERG, J.; JÄGGI, M.; BEER, H.; BAEHRIE, H. (Eds.). LSC 2008 Advances in Liquid Scintillation Spectrometry. [S.I.]: Radiocarbon, p. 59-69.

ZAPATA-GARCÍA, D.; LLAURADÓ, M.; RAURET, G. (2012) The implications of particle energy and acidic media on gross alpha and gross beta determination using liquid scintillation. Applied and Radiation Isotopy, v. 70, p. 705-711.

ZORER, O.S. \& ŞAHAN, T.J. (2011) The concentration of ${ }^{238} \mathrm{U}$ and the levels of gross radioactivity in surface waters of the Van Lake (Turkey). Journal of Radioanalytical and Nuclear Chemistry, v. 288, p. 417-421. 